\title{
Brogaard and Salerno on antirealism and the conditional fallacy
}

\author{
Luca Moretti \\ Centre for Time \\ University of Sydney \\ luca.moretti@arts.usyd.edu.au
}

\begin{abstract}
Brogaard and Salerno 2005 have argued that antirealism resting on a counterfactual analysis of truth is flawed because it commits a conditional fallacy by entailing the absurdity that there is necessarily an epistemic agent. Brogaard and Salerno's argument relies on a formal proof built upon the criticism of two parallel proofs given by Plantinga 1982 and Rea 2000. If this argument were conclusive, antirealism resting on a counterfactual analysis of truth should probably be abandoned. I argue however that the antirealist is not committed to a controversial reading of counterfactuals presupposed in Brogaard and Salerno's proof, and that the antirealist can in principle adopt an alternative reading that makes this proof invalid. My conclusion is that no reductio of antirealism resting on a counterfactual analysis of truth has yet been provided.
\end{abstract}

\section{Alethic antirealism and the conditional fallacy: the state of play}

Global alethic antirealists analyse the notion of truth in terms of the biconditionals instantiated by the following schema:

$$
(\mathrm{AR}) \square(\mathrm{T}[P] \leftrightarrow(\mathrm{Q}(P) \square \rightarrow \mathrm{R}(P))) .
$$

Where $P$ is a placeholder for statements, $\mathrm{T}[P]$ means that it is true that $P, \mathrm{Q}(P)$ means that epistemic conditions are ideal (or sufficiently good) for determining whether $P, \mathrm{R}(P)$ means that it is rationally believed that $P$. Furthermore, $\square$ is the necessity operator, $\leftrightarrow$ is the material biconditional and $\square \rightarrow$ is the counterfactual conditional. AR says that, necessarily, it is true that $P$ if and only if, if 
epistemic conditions were ideal (or sufficiently good) for determining whether $P$, it would rationally be believed that $P$.

Brogaard and Salerno 2005 argue that antirealism resting on AR is flawed because it commits a conditional fallacy by entailing the absurdity that, necessarily, epistemic conditions are ideal for determining whether some statement is true, which implies that there is necessarily an epistemic agent. Brogaard and Salerno's argument relies on a formal proof built upon the criticism of two parallel proofs given by Plantinga 1982 and Rea 2000. ${ }^{1}$ Precisely, both demonstrations are argued to be questionable because appealing to principles valid only in classical logic that the antirealist may reject in favour of intuitionistic principles (cf. Brogaard and Salerno 2005: 126 and 130). Additionally, Plantinga's original proof and an intuitionistically respectable reformulation of it are found to lack in generality (cf.: 124-126). For they can successfully apply only to Peircean versions of antirealism, which require a commitment to the existence of one single epistemic situation (e.g. "the end of inquiry") appropriate to establish the truth of any statements. Such positions are argued to be unattractive and implausible on their own (cf.: 128-129).

By contrast, Brogaard and Salerno's novel argument against antirealism is quite general, as it targets both Peircean antirealism and non-Peircean antirealism, which allows statements to have individual truth conditions (cf.: 135-136). Brogaard and Salerno also emphasize that their proof relies on only 'exceedingly weak logical resources' (137) that the antirealist cannot but accept. Finally, Brogaard and Salerno make a case that 'an [antirealist] attempt to reinterpret $\square \rightarrow$ in a way that blocks the conditional fallacy will ... carry with it unacceptable consequences of its own' (ibid.). Brogaard and Salerno are thus persuaded that 'the counterfactual analysis of truth as epistemic cannot avoid the conditional fallacy, not without a massive revisionism of our logical resources' (ibid.) They believe that 'the counterfactual analysis of truth must fall prey to some counterintuitive consequence - viz., that an epistemic agent must exist' (ibid.). 
I find Brogaard and Salerno's criticism of Plantinga's and Rea's proofs quite persuasive and I will not question it, while I will take issue with Brogaard and Salerno's new argument. If this argument were conclusive, antirealism resting on a counterfactual analysis of truth should probably be abandoned. I argue however that the antirealist is not committed to the controversial reading of counterfactuals presupposed in Brogaard and Salerno's proof, according to which counterfactuals with impossible antecedent are vacuously true. I show that, despite Brogaard and Salerno's conviction, the antirealist can in principle interpret $\square \rightarrow$ in an alternative way that blocks the conditional fallacy without making any unacceptable assumption. The antirealist can thus reject Brogaard and Salerno's proof as invalid.

In more detail, in Sect 2, I introduce the notion of a conditional fallacy and present Brogaard and Salerno's argument against alethic antirealism. In Sect 3, I argue that the antirealist can reject Brogaard and Salerno's formal proof by appealing to an alternative analysis of counterfactuals, and I outline Brogaard and Salerno's anticipated reply to this argument. In Sect 4, I show that Brogaard and Salerno's anticipated reply is unsuccessful. In Sect 5, I draw the conclusions of the paper.

\section{The conditional fallacy and Brogaard and Salerno's proof}

Suppose a given notion is analysed in the terms of the biconditionals instantiated by the following schema:

$$
\square(P \leftrightarrow(Q \square \rightarrow R)),
$$

where $P, Q$ and $R$ are placeholders for statements, $P$ stands for the analysandum and $(Q \square \rightarrow R)$ for the analysans. The problem of the conditional fallacy arises if some substitutions for $P$ and $Q$ are not logically independent. In such cases, counterintuitive modal consequences can be derived: for instance, statements that are obviously contingent may turn out to be necessarily true or necessarily false (cf. Brogaard and Salerno 2005: 134-135). In particular, Brogaard and Salerno focus on the 
following meta-statement, which - they contend - proves true on very weak logical assumptions (where $\rightarrow$ is the material conditional):

(CF) $\{\square(P \leftrightarrow(Q \square \rightarrow R)), \square(Q \rightarrow P)\}$ entails $\square P$.

They argue that each instance of AR produces the fulfilment of $\square(P \leftrightarrow(Q \square \rightarrow R))$, and that, for some of these instances, $\square(Q \rightarrow P)$ will also be satisfied. Therefore, the antirealist ought to deduce, in certain cases, instances of $\square P$. This would commit the antirealist to absurdity. (Cf.: 131).

Brogaard and Salerno give just one example of this (cf.: 135-136). Consider the instance of $\square(P$ $\leftrightarrow(Q \square \rightarrow R))$ in which:

$P=$ 'epistemic conditions are ideal for determining whether some statement is true';

$Q=$ 'epistemic conditions are ideal for determining whether [epistemic conditions are ideal for determining whether some statement is true]';

$R=$ 'it is rationally believed that [epistemic conditions are ideal for determining whether some statement is true]'.

This instance of $\square(P \leftrightarrow(Q \square \rightarrow R))$ logically follows from the instance of AR with $P=$ 'epistemic conditions are ideal for determining whether some statement is true' and the necessitation of the Equivalence Schema. ${ }^{2}$ The antirealist is thus committed to it. But the antirealist is also committed to the correlated instance of $\square(Q \rightarrow P)$. As, in this interpretation of $P$ and $Q, Q$ entails $P$ through the necessitation of the Equivalence Schema. ${ }^{3}$ Given CF, the antirealist must then derive that $\square P-$ namely, that, necessarily, epistemic conditions are ideal for determining whether some statement is true. These epistemic conditions 'include the existence of a properly placed epistemic agent. So, necessarily, there is an epistemic agent' (135). 
The success of this supposed reductio of alethic antirealism crucially depends on whether the antirealist is committed to CF. Brogaard and Salerno seek to prove CF in the modal system $\mathbf{S} 4$ extended with logical resources for counterfactuals. $\mathbf{S 4}$ is characterized by axiom schema M, i.e. $\square Q \rightarrow Q$, and axiom schema 4, i.e. $\square Q \rightarrow \square \square$. Although axiom $\mathrm{M}$ allows for the formal characterization of features of modal notions that are largely uncontroversial, axiom 4 has been criticized. N. Salmon 1989 and 1993 has for example delivered a sorites argument to the effect that certain instances of axiom 4 are intuitively false. As a result, though the antirealist is plausibly committed to axiom M, her commitment to axiom 4 might be questioned. Brogaard and Salerno do not consider this potential flaw of their reductio: they appear to accept axiom 4 as unproblematic. ${ }^{45}$ Therefore, one line of defence for the antirealist might be that of trying to reject Brogaard and Salerno's proof via challenging axiom 4's validity. ${ }^{6}$ In this paper, I defend however a more conservative and prudent strategy that may be pursued by the antirealist. ${ }^{7}$ I will show that the antirealist can dismiss Brogaard and Salerno's proof even while accepting axiom 4. The antirealist can thus comfortably retain much of our logical background without falling afoul of this attempted reductio.

The proof of $\mathrm{CF}$ requires the following two additional axiom schemata for counterfactuals (where $\wedge$ is logical conjunction):

$$
\begin{aligned}
& \text { (A1) }(Q \wedge(Q \square \rightarrow R)) \rightarrow R ; \\
& \text { (A2) } \square(Q \rightarrow R) \rightarrow(Q \square \rightarrow R) .
\end{aligned}
$$

(Strictly speaking, Brogaard and Salerno formulate A1 and A2 as inference rules, but turning these rules into schemata will simplify my discussion). Axiom A1, which can be formulated as a special modus ponens for counterfactuals, has strong intuitive appeal. The antirealist could reject A1 with difficulty. ${ }^{8}$ Axiom A2 corresponds to the rule that a counterfactual can logically be inferred from 
the corresponding strict conditional. A2 is much more dubious and controversial than A1. The antirealist can straightforwardly question its validity (more on this in the next section).

Here is Brogaard and Salerno's proof (where the inference rules corresponding to A1 and A2 have been replaced with these two axiom schemata) (cf. 2005: 131):

$$
\begin{aligned}
& \text { 1. } \square(P \leftrightarrow(Q \square \rightarrow R)), \square(Q \rightarrow P) \\
& \text { 2. } P \leftrightarrow(Q \square \rightarrow R), Q \rightarrow P \\
& \text { 3. } Q \\
& \text { 4. } Q \square \rightarrow R \\
& \text { 5. } R
\end{aligned}
$$$$
\text { 6. } Q \rightarrow R
$$$$
\text { 7. } \square(Q \rightarrow R)
$$$$
\text { 8. } Q \square \rightarrow R
$$$$
\text { 9. } P
$$$$
\text { 10. } \square(Q \rightarrow R)
$$$$
\text { 11. } \square(Q \square \rightarrow R)
$$$$
\text { 12. } \square P
$$

QED.

\section{(Assumptions)}

(From 1 and axiom M, by modus ponens)

(Assumption, for $\rightarrow$ introduction)

(From 2 and 3, by modus ponens)

(From 3, 4, and A1, by ^introduction and modus ponens)

(From 3-5, by $\rightarrow$ introduction)

(From 1 and 2-6, by closure)

(From 7 and A2, by modus ponens)

(From 2 and 8, by modus ponens)

(From 7 and axiom 4, by modus ponens)

(From 7, 8 and 10, by closure)

(From 1, 2, 8, 9 and 11, by closure)

$Q \square \rightarrow R$, on line 4, follows from $P \leftrightarrow(Q \square \rightarrow R)$ and $Q \rightarrow P$, on line 2, and from $Q$, on line 3, because $Q$ and $Q \rightarrow P$ entail $P$ by modus ponens, and $P$ and $P \leftrightarrow(Q \square \rightarrow R)$ entail $Q \square \rightarrow R$ by modus ponens. ${ }^{9}$ On line 7, we can necessitate $Q \rightarrow R$ by closure (i.e. the rule that necessities entail necessities) because $Q \rightarrow R$ follows from $P \leftrightarrow(Q \square \rightarrow R)$ and $Q \rightarrow P$, which are assumed on line 1 to be both necessary. ${ }^{10}$ On line 11 , we can necessitate $Q \square \rightarrow R$ by closure because $Q \square \rightarrow R$ follows from $\square(Q \rightarrow R)$, which is shown to be necessary on line 10 . On line 12 , we can necessitate $P$ by closure because $P$ follows from $P \leftrightarrow(Q \square \rightarrow R)$, which is assumed to be necessary on line 1 , and from $Q \square \rightarrow R$, which is shown to be necessary on line 11 . 
Brogaard and Salerno concede that 'the antirealist's commitment to classical logic is doubtful' (130) but at the same time stress that they have proven CF 'without principles that are exclusively classical' (136), so that 'the proof is valid in intuitionistic (and even minimal) logic' (ibid.). What Brogaard and Salerno plausibly mean is that their proof is valid in a suitable intuitionistic modal logic extended with axioms A1 and A2 for counterfactuals. To make my criticism more precise, I will make this non-classical logic explicit.

Intuitionistic modal logic has recently been systematized into a family of logics each of which corresponds to a member of a parallel family of classical modal logics (see for instance Fisher Servi 1980 and 1984, Simpson 1994: §3.2-3.4, and Gabbay et al. 2003: 3.11 and $\S 10) .{ }^{11}$ IK is the weakest intuitionistic modal logic of the family. IK is conservative over intuitionistic propositional logic, contains all substitution instances of theorems of the latter logic and is closed under modus ponens. ${ }^{12}$ Furthermore, the mere addition of the axiom schema $P \vee \neg P$ (where $\vee$ is logical disjunction and $\neg$ is logical negation) to IK produces the weakest classical modal system $\mathbf{K}$. Each member of the ordered triple of intuitionistic modal logics $\mathscr{I}=<\mathbf{I T}$, IS4, IS5 $>$, resulting from adding further axiom schemata to IK, corresponds, in the same order, to a member of the ordered triple of classical modal logics $\mathscr{C}=<\mathbf{T}, \mathbf{S 4}, \mathbf{S 5}>$, resulting from adding the same axiom schemata to K. Each member of $\mathscr{I}$ is thus characterized by exactly the same additional axiom schemata that characterize its counterpart in $\mathscr{C}$, and the addition of $P \vee \neg P$ to any member of $\mathscr{J}$ yields its classical

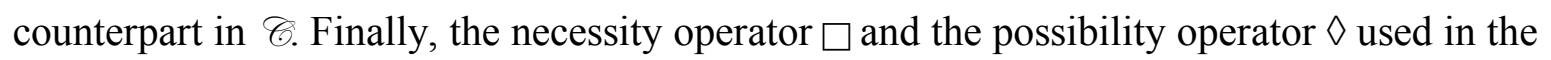
intuitionistic logics of $\mathscr{N}\{\mathbf{I K}\}$ reflect the behaviour of, respectively, the intuitionistic quantifiers $\forall$ and $\exists$, in the same way as the modal operator $\square$ and $\diamond$ used in the classical logics of $\mathscr{C} \cup\{\mathbf{K}\}$ simulate the classical quantifiers $\forall$ and $\exists$ (cf. Gabbay et al. 2003: 188). An expected consequence is that the intuitionistic $\square$ and $\diamond$ are not interdefinable (in the sense it does not hold valid that $\diamond P \leftrightarrow$ $\neg \square \neg P) .^{1314}$ 
The logics of $\mathscr{N}\{\mathbf{I K}\}$ are provided with a possible world semantics. Precisely, these logics are sound and complete with respect to certain classes of frames of the form $<\mathrm{W}, \leq, \mathrm{R}>$, where $\mathrm{W}$ is a set of possible worlds partially ordered by $\leq$, and $\mathrm{R}$ is a binary relation - the accessibility relation defined on W. These frames combine aspects of Kripke's frames for classical modal logic and Kripke’s frames for intuitionistic predicate logic (cf. Simpson 1994: $\$ 3.3$ and Gabbay et al. 2003: $\S 3.11$ and $\S 10) .{ }^{15}$

As IS4 is just characterized by axioms M and 4, which are necessary to Brogaard and Salerno's demonstration, ${ }^{16}$ IS4 $\cup\{\mathrm{A} 1, \mathrm{~A} 2\}$ appears suitable to carrying out this proof. ${ }^{17}$ Indeed, the logic IS4 $\cup\{\mathrm{A} 1, \mathrm{~A} 2\}$ does validate this proof. Brogaard and Salerno 2005 claim that the counterfactual analysis of truth as epistemic cannot avoid the conditional fallacy, 'not without a massive revisionism of our logical resources' (137). This claim appears simply false. I will show that the antirealist can comfortably accept IS4, and even IS4 $\cup\{\mathrm{A} 1\}$, while rejecting the dubious A2. Without A2, no conditional fallacy will probably result. Assuming - more implausibly - that the antirealist endorses classical modal logic, all my arguments can easily be reformulated, with only negligible changes, to show that the antirealist can accept $\mathbf{S 4}$, and even $\mathbf{S} 4 \cup\{\mathrm{A} 1\}$, while dropping the dubious A2.

\section{The antirealist's rejoinder and Brogaard and Salerno's reply}

The inference of line 8 from line 7 consists in deducing the counterfactual $Q \square \rightarrow R$ from the strict conditional $\square(Q \rightarrow R)$. This inference is licensed by modus ponens and A2. If A2 were dropped, line 8 could not be inferred from line 7, and Brogaard and Salerno's proof of CF would be incorrect. Can the antirealist reject $\mathrm{A} 2$ ? Notice that $\mathbf{I K} \cup\{\mathrm{A} 2\}$ validates the schema $\neg \vee Q \rightarrow(Q \square \rightarrow R)$, which expresses the thesis that counterpossibles (i.e. counterfactuals with impossible antecedent) are vacuously true. ${ }^{18}$ This schema is not validated by $\mathbf{I K}$ alone (neither by $\mathbf{I K} \cup\{\mathrm{A} 1\}$ ). As the antirealist who accepts intuitionistic logic is plausibly committed to IK, if the antirealist rejected 
$\neg \vee Q \rightarrow(Q \square \rightarrow R)$ as invalid, she should also reject A2 as invalid. This would make the proof of

$\mathrm{CF}$ incorrect. The crucial question is, therefore, whether the antirealist can reject $\neg \vee Q \rightarrow(Q \square \rightarrow$ $R$ ), that is, the claim that counterpossibles are vacuously true.

Lewis 1973 proposes that a counterfactual $Q \square \rightarrow R$ is true at a possible world $w$ if and only if (a) $Q$ is impossible at $w$ or (b) in the possible worlds where $Q$ is true and which are closest to $w$ (i.e. in those worlds that resemble $w$ as much as $Q$ 's truth permits it), $R$ is true (cf.: 16). Accepting clause (a) is the same as accepting that counterpossibles are vacuously true. While clause (b) has intuitive appeal and is generally accepted as a sufficient condition for the truth of a counterfactual, this is hardly the case with clause (a), which is extremely contentious. Lewis 1973 himself has admitted that his 'reasons [to accept (a)] are less than decisive' (25). Among the numerous critics of (a), see for instance: Yagisawa 1988, Zagzebski 1990, Read (1995: 90-91), Mares 1997, Nolan 1997, Priest 1997 and Goodman 2004. An important reason why philosophers question clause (a) is that although certain counterpossibles appear intuitively true, other counterpossibles appear intuitively false (cf. Mares 1997: 517 and Goodman 2004: 35-36). For example, the following counterpossibles seem true:

If Katie were to square the circle, we would be surprised;

If water were an element, it could not be broken into hydrogen and oxygen.

While these counterpossibles appear false:

If Katie were to square the circle, we would not be surprised;

If water were an element, it could be broken into hydrogen and oxygen.

Indeed, there is no general agreement on the correct semantic analysis of counterpossibles, and it is not evident that the alethic antirealist as such is committed to any specific view in this controversial issue. But if the antirealist is not committed to (a), she can reject it together with $\neg \vee Q$ 
$\rightarrow(Q \square \rightarrow R)$. The consequence is that A2 will also be dropped. The antirealist can thus reject Brogaard and Salerno's proof of CF as incorrect by accepting IS4, and even IS4 $\cup\{$ A1 $\}$, while dropping A2.

Brogaard and Salerno 2005 are well aware of this possible counterargument (cf.: 126-127 and 136-137), but they believe it cannot succeed. If the antirealist drops (a), the antirealist should endorse an alternative analysis of counterpossibles. Brogaard and Salerno examine two possibilities. The first analysis, considered also by Lewis (1973: 25), 'treats a counterfactual as false when it embeds an impossible antecedent' (2005: 126). 'Alternatively, and more plausibly, counterfactuals with necessary false antecedents are to be treated as sometimes true, sometimes false' (127). The second alternative appears more plausible at least because it complies with the fact that certain counterpossibles are intuitively true and others intuitively false. Brogaard and Salerno contend that should the antirealist drop (a) to accept either alternative reading of counterpossibles, she would not escape the conditional fallacy. For, to block the deduction of line 8 from line 7 in the proof of CF namely, of $Q \square \rightarrow R$ from $\square(Q \rightarrow R$ ) - the antirealist should additionally assume that $\neg \diamond Q$ (cf.: 137 and note 13: 138). Considering the interpretation of $Q$ that elicits the conditional fallacy, this means that the antirealist should assume that $\neg \diamond \mathrm{Q}(\mathrm{P})$. Where $\mathrm{Q}(\mathrm{P})$ means that epistemic conditions are ideal for determining whether $\mathrm{P}$, and $\mathrm{P}$ means that epistemic conditions are ideal for determining whether some statement is true. Brogaard and Salerno are persuaded that if this additional condition is not satisfied, their reductio of antirealism will still go through even if (a) and A2 are dropped; and they argue that this additional condition cannot be satisfied on either of the two alternative readings of counterpossibles. For, in both cases, and for different reasons, it turns out that $\neg \diamond \mathrm{Q}(\mathrm{P})$ is false. (Cf.: 136-137 and note 13: 138).

In the remainder of my paper, I will criticize the specific argument by Brogaard and Salerno that targets the appeal to the sometimes-true-sometimes-false reading of counterpossibles - which is the more plausible of the two alternatives they have individuated. Against Brogaard and Salerno, I will 
argue that if the antirealist drops (a), and so A2, to endorse this alternative reading of counterfactuals, this move will suffice to prevent the conditional fallacy. Despite Brogaard and Salerno's conviction, the antirealist does not need to assume that $\neg \diamond \mathrm{Q}(\mathrm{P})$.

\section{Brogaard and Salerno's reductio of antirealism is ineffective}

Brogaard and Salerno 2005 believe that the mere endorsement of the view that some counterpossibles are true and some false does not suffice to disable their reductio. To block it:

It must be also argued in a principled way that $\mathrm{Q}(\mathrm{P})$ is impossible whenever $\mathrm{P}$ is a statement that triggers a conditional fallacy. For example, when $\mathrm{P}$ is the statement 'epistemic condition are ideal for some statement', it must be argued that it is impossible for conditions to be ideal for the proper evaluation of 'conditions are ideal for some statements'. It is difficult to see how such an argument would go, since often enough it is the case that conditions are in fact ideal/sufficiently good for the evaluations of this statement. Conditions are currently sufficiently good for the evaluation of whether conditions are sufficiently good for some statement. To show that $\mathrm{Q}(\mathrm{P})$ is impossible will then be to show too much. An attempt to reinterpret $\square \rightarrow$ in a way that blocks the conditional fallacy will then carry with it unacceptable consequences of its own (137. I have slightly changed the original notations).

Although Brogaard and Salerno do not spell out the ideal conditions for determining whether 'epistemic condition are ideal for determining whether some statement is true', it seems intuitively plausible that an acceptable analysis of them should entail that such conditions are often fulfilled. And, if $\mathrm{Q}(\mathrm{P})$ is true, given axiom $\mathrm{M}, \mathrm{Q}(\mathrm{P})$ is also possible. Therefore, Brogaard and Salerno are very probably right in concluding that the antirealist who endorses the sometimes-true-sometimesfalse reading of counterpossibles cannot succeed in arguing that $\neg \diamond \mathrm{Q}(\mathrm{P})$. 
But why do Brogaard and Salerno believe that, to block their reductio, the antirealist needs to argue that $\mathrm{Q}(\mathrm{P})$ is impossible whenever $\mathrm{P}$ is a statement that triggers a conditional fallacy? Unfortunately, they do not clarify this crucial point. A possible answer is that Brogaard and Salerno are simply mistaken. Let us examine once again the strategy for the antirealist that Brogaard and Salerno themselves have suggest (cf.: 126 and 137). The antirealist will endorse the sometimestrue-sometimes-false reading of counterpossibles; she will thus reject the schema $\neg \vee Q \rightarrow(Q \square \rightarrow$ $R$ ), which expresses the thesis that all counterpossibles are vacuously true. If the antirealist rejects $\neg \vartheta Q \rightarrow(Q \square \rightarrow R)$, she ought also to drop A2 (i.e. $\square(Q \rightarrow R) \rightarrow(Q \square \rightarrow R))$. But then, the antirealist will be deprived of the essential axiom schema (or of the corresponding rule) to deduce a counterfactual from the relative strict conditional. Consequently, the logical deduction of line 8 from line 7 - namely, of $Q \square \rightarrow R$ from $\square(Q \rightarrow R)$ - in the proof of CF will turn out to be unjustified for the antirealist. Therefore, this very proof will be invalid for her. In conclusion, the antirealist who accepts the sometimes-true-sometimes-false reading of counterpossibles needs no further assumption to block Brogaard and Salerno's reductio.

A more charitable and interesting explanation of Brogaard and Salerno's conviction is that they are persuaded that a simple variant of their reductio will go through if $\diamond \mathrm{Q}(\mathrm{P})$ is true. This might be the reason why they believe that, to block the conditional fallacy, the antirealist needs to argue that $\neg \diamond \mathrm{Q}(\mathrm{P})$ whenever $\mathrm{P}$ is a statement that triggers a conditional fallacy. If this is what Brogaard and Salerno believe, their belief is very probably false. The reminder of this section is devoted to argue that, in all probability, there is no simple variant of Brogaard and Salerno's reductio that goes through when $\diamond \mathrm{Q}(\mathrm{P})$ is true.

Brogaard and Salerno might believe that, as the antirealist is committed to $\diamond \mathrm{Q}(\mathrm{P})$, the antirealist should assume $\diamond Q$ among her premises (as $Q$ has been interpreted as $\mathrm{Q}(\mathrm{P})$ ), and that this would allow her to deduce $Q \square \rightarrow R$ from $\square(Q \rightarrow R)$ and $\diamond Q$, on the basis of an appropriate axiom schema (or inference rule). For example this axiom schema: 
(A3) $(\square(Q \rightarrow R) \wedge \diamond Q) \rightarrow(Q \square \rightarrow R)$.

As the intuitive validity of A3 appears to depend on the sole clause (b) of Lewis' analysis of counterfactuals, which is strongly plausible, the antirealist is probably committed to this axiom schema. ${ }^{19}$ Consequently, if the antirealist accepts both $\diamond Q$ and $\square(Q \rightarrow R)$, she can correctly infer $Q$ $\square \rightarrow R$, by $\mathrm{A} 3$, $\wedge$ introduction and modus ponens.

To try to make their reductio of antirealism operative, Brogaard and Salerno might thus appeal to a simple variant of $\mathrm{CF}$ that includes the additional premise $\diamond \mathrm{Q}$. Precisely:

(CF1) $\{\square(P \leftrightarrow(Q \square \rightarrow R)), \square(Q \rightarrow P), \diamond Q\}$ entails $\square P$.

As the antirealist is probably committed to $\mathrm{A} 3$, it would seem reasonable to use this axiom schema, instead of A2, in the proof of CF1. The problem is that it will not work. Indeed, no proof of CF1 appears at hand in IS4 $\cup\{\mathrm{A} 1, \mathrm{~A} 3\}$.

Here is an attempted proof of CF1 consisting in a variation of CF's original proof that makes use of $\mathrm{A} 3$ rather than $\mathrm{A} 2$ :

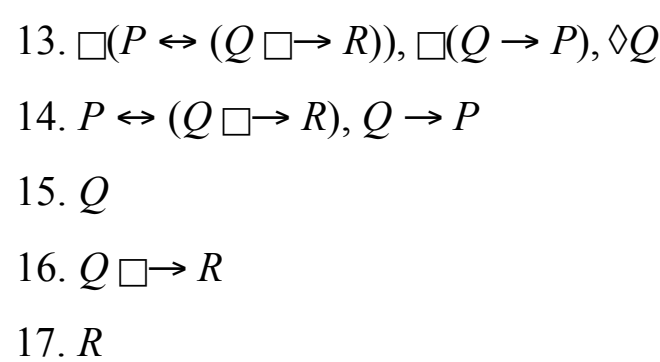

18. $Q \rightarrow R$

19. $\square(Q \rightarrow R)$

20. $Q \square \rightarrow R$

21. $P$

22. $\square(Q \rightarrow R)$
(Assumptions)

(From 13 and axiom M, by modus ponens)

(Assumption, for $\rightarrow$ introduction)

(From 14 and 15, by modus ponens)

(From 15, 16, and A1, by $\wedge$ introduction and modus ponens)

(From 15-17, by $\rightarrow$ introduction)

(From 13 and 14-18, by closure)

(From 13 and 19, by $\wedge$-introduction and A3)

(From 14 and 20, by modus ponens)

(From 19 and axiom 4, by modus ponens) 
23. $\square(Q \square \rightarrow R)$

24. $\square P$

QED.
(From 13, 19 and 22, by closure)

(From 13, 14, 20, 21 and 23, by closure)

This proof is invalid. On line 23, we cannot necessitate $Q \square \rightarrow R$ by closure because $Q \square \rightarrow R$ follows now from two schemata: $\square(Q \rightarrow R)$, on line 19, and $\diamond Q$, on line 13. Of these two schemata, only $\square(Q \rightarrow R)$, but not $\diamond Q$, is necessary $(\square(Q \rightarrow R)$ is shown to be necessary on line 22). It is not apparent that any correct proof of CF1 is possible at all in IS4 $\cup\{\mathrm{A} 1, \mathrm{~A} 3\}$.

Replacing the premise $\diamond Q$, on line 13, with the stronger $\square \diamond Q$ will produce a valid proof. But it is not evident that the antirealist who accepts the view that counterpossibles are sometimes true and sometimes false is committed to $\square \diamond Q$ when $Q$ means 'epistemic conditions are ideal for determining whether [epistemic conditions are ideal for determining whether some statement is true]'. Brogaard and Salerno have given no reason to support this claim (which they do not even discuss in their paper). Consequently, the antirealist can plausibly reject the proof including the stronger premise $\square \diamond Q$ as unsound when the proof is used in a reductio of antirealism.

Indeed, the antirealist will derive $\square \diamond Q$ from the original premise $\diamond Q$ in a modal logic as strong as IS5, as the latter validates axiom 5, i.e. $\diamond Q \rightarrow \square \diamond Q .{ }^{20}$ If the antirealist accepted IS5, A1 and A3, she would be committed to a proof of CF1 carried out in IS5 $\cup\{\mathrm{A} 1, \mathrm{~A} 3\}$. But why should the antirealist be committed to a modal system as strong as IS5? (Notice that Brogaard and Salerno would probably doubt of such a commitment, as they seek to carry out their reductio in an elementary extension of S4). An immediate motive of concern about the acceptability of IS5 which might worry also the antirealist - is that, given the strength of IS5, certain intuitive modal distinctions cannot be drawn in this logic. There does seem to be a difference between to say, for example, that something is possible, and saying that something is necessarily possible. Yet these two statements are equivalent in IS5, which validates both $\diamond Q \rightarrow \square \diamond Q$ and $\square \diamond Q \rightarrow \diamond Q .{ }^{21}$ More importantly, as Armstrong (1989: 62-63) and others have shown, substantial reasons to refuse 
axiom 5 will result if a combinatorialist theory of possibility is accepted - namely, a metaphysical picture according to which, roughly, possible worlds are nothing but rearrangements or recombinations of contingent elements - e.g. individuals and properties - existing in the actual world. $^{22}$ In contemporary metaphysics there is no agreement on the correct analysis of possibility: the debate on this very complex topic is still open and on going. Yet, many philosophers find combinatorialism attractive because of its ontological parsimony and because it harmonizes with actualism and naturalism. Combinatorialism is certainly affected by some difficulties, but this is true of any other interesting theory of possibility. ${ }^{23}$ The alethic antirealist appears prima facie entitled to endorse some version of combinatorialism (for instance, one according to which the contingent recombining items of the actual world - e.g. individuals and properties - are minddependent). But then, dropping axiom 5 is no insurmountable problem for her. The alethic antirealist does not appear committed to a modal logic as strong as IS5.

It might be thought that the difficulties we are facing depend on relying on A3, while Brogaard and Salerno would appeal to a more suitable axiom schema (or a corresponding inference rule) in a revised version of $\mathrm{CF}^{24}$ For instance this:

$$
\text { (A4) } \diamond Q \rightarrow \square(\square(Q \rightarrow R) \rightarrow(Q \square \rightarrow R)) .^{25}
$$

Appealing to A4, rather than to A3, does result in a correct demonstration of CF1 in IS4 $\cup\{\mathrm{A} 1$, A4\}. The problem is that this strong principle does not seem to have intuitive appeal. A4 is typically not included among the general principles that govern counterfactuals (cf. for instance Lewis 1973: 132). Furthermore, A4 does not appear validated by the sole clause (b) of Lewis' analysis of counterfactuals. Why should the antirealist accept A4? Indeed, A4 is demonstrable in IS5 $\cup\{\mathrm{A} 3\}$ (and thus in IS5 $\cup\{\mathrm{A} 1, \mathrm{~A} 3\}$ ). But this does not help. For the antirealist's commitment to a modal logic as strong as IS5 is, as I have indicated, dubious. 
It might be suggested that all our difficulties arise because we want to justify the possibility of inferring a counterfactual from a strict conditional (i.e. the move from line 7 to line 8 in $\mathrm{CF}$, and from line 19 to line 20 in CF1) by appealing to axiom schemata like A2, A3 or A4, while Brogaard and Salerno would appeal to no such principles. They would rather provide a reformulation of CF more sophisticated than CF1, which would allow inferring a counterfactual from the relative strict conditional even in IS4 $\cup\{\mathrm{A} 1\}$. I believe, however, that this alternative strategy does not fare any better.

Consider for instance a reformulation of CF that makes use of the additional premise $\square(Q \rightarrow R)$ $\rightarrow(Q \square \rightarrow R)$ rather than of $\diamond Q$. Precisely:

(CF2) $\{\square(P \leftrightarrow(Q \square \rightarrow R)), \square(Q \rightarrow P), \square(Q \rightarrow R) \rightarrow(Q \square \rightarrow R)\}$ entails $\square P$.

As the antirealist is plausibly committed to A3 (i.e. $(\square(Q \rightarrow R) \wedge \diamond Q) \rightarrow(Q \square \rightarrow R)$ ), the antirealist will probably accept the additional premise of CF2, $\square(Q \rightarrow R) \rightarrow(Q \square \rightarrow R)$, if she accepts $\vee Q$. For $\square(Q \rightarrow R) \rightarrow(Q \square \rightarrow R)$ follows from $\diamond Q$ in IK $\cup\{\mathrm{A} 3\}$. In a proof of CF2, we could deduce $Q$ $\square \rightarrow R$ from $\square(Q \rightarrow R)$ by the mere appeal to $\square(Q \rightarrow R) \rightarrow(Q \square \rightarrow R)$ and modus ponens. No additional inference rule or axiom schema, such as A2, A3 or A4, is necessary for this deduction. The problem is that it can be shown that no proof of CF2 is possible in IS4 $\cup\{\mathrm{A} 1\} .{ }^{26}$

If the premise $\square(Q \rightarrow R) \rightarrow(Q \square \rightarrow R)$ were changed into $\square(\square(Q \rightarrow R) \rightarrow(Q \square \rightarrow R)),{ }^{27}$ a proof of CF2 would go through in IS4 $\cup\{\mathrm{A} 1\}$. But it is unclear why the antirealist who claims that counterpossibles are sometimes true and sometimes false should be committed to such a strong premise when $Q$ and $R$ are interpreted, respectively, as $\mathrm{Q}(\mathrm{P})$ and $\mathrm{R}(\mathrm{P})$. Namely, when $\mathrm{Q}(\mathrm{P})$ means that epistemic conditions are ideal for determining whether $\mathrm{P}, \mathrm{R}(\mathrm{P})$ means that it is rationally believed that $\mathrm{P}$, and $\mathrm{P}$ means that epistemic conditions are ideal for determining whether some statement is true. Brogaard and Salerno could substantiate this commitment if they were able to show that the antirealist is committed to $\square \diamond \mathrm{Q}(\mathrm{P})$. For $\square \diamond Q$ entails $\square(\square(Q \rightarrow R) \rightarrow(Q \square \rightarrow R))$ in IK 
$\cup\{\mathrm{A} 3\}$, and $\mathrm{A} 3$ would seem to be intuitionistically valid. But, again, why should the antirealist be committed to $\square \diamond \mathrm{Q}(\mathrm{P})$ ?

At this point, one might complain that the background logic for counterfactual I have conceded to the antirealist - i.e. IS4 $\cup\{\mathrm{A} 1, \mathrm{~A} 3\}$ - is exceedingly weak. For instance, it could easily be shown that this system does not even validate the intuitively plausible principle $Q \square \rightarrow Q$. The suspicion might be that if further reasonable schemata or rules specific for counterfactuals were added, a reductio of antirealism might obtain even without accepting the questionable A2. ${ }^{28}$ This might be true. Although the mere addition of an axiom schema as trivial as $Q \square \rightarrow Q$ would seem to settle none of the problems of the attempted proofs of a conditional fallacy considered so far, the introduction of more sophisticated axiom schemata might perhaps do. Investigating whether this is actually the case is however beyond the scope of this paper. I have limited myself to consider the very same resources for counterfactuals of which Brogaard and Salerno appear to avail the antirealist. ${ }^{29}$ My specific purpose is criticizing the proof that Brogaard and Salerno have explicitly given and some simple variants of it - those that Brogaard and Salerno might implicitly have in mind, according to my more charitable interpretation of their argument. My paper aims to show that no proof that the antirealist commits a conditional fallacy has been provided, and not that such a proof is impossible.

The moral to be learned is that Brogaard and Salerno have not proven that their reductio will succeed if the antirealist who accepts a counterfactual analysis of truth also believes that counterpossibles are sometimes true and sometimes false. If the antirealist accepts this semantical view, Brogaard and Salerno's proof will not go through and, probably enough, no simple variation of it will work either. To counter Brogaard and Salerno's reductio, the antirealist does not need to argue that $\neg \diamond \mathrm{Q}(\mathrm{P})$. Instead, she should merely emphasize that, on her reading of counterpossibles, the proof of CF given by Brogaard and Salerno is invalid, for the inference from line 7 to line 8 is unjustified, and that no alternative proof which the antirealist is committed to appears available. 


\section{Conclusion: no proof that alethic antirealism commits a conditional fallacy has yet been}

\section{delivered}

Brogaard and Salerno 2005 have made a case that global alethic antirealism resting on a counterfactual analysis of truth is flawed because commits a conditional fallacy by entailing the absurdity that there is necessarily an epistemic agent. This argument is general, as it targets different forms of antirealism all at once, and it does not depends on principles of classical logic that the antirealist can reject. In these respects, Brogaard and Salerno's case is less questionable than parallel arguments made by Plantinga and Rea.

I have argued however that the antirealist can block Brogaard and Salerno's reductio with a simple move that involves no drastic revision of intuitionistic modal logic (or of classical modal logic, should the antirealist endorse it). The crucial demonstration which Brogaard and Salerno's argument relies on takes for granted the controversial thesis that counterfactuals with impossible antecedent are vacuously true. I have argued that the antirealist can in principle reject this thesis by embracing the alternative view that counterfactuals with impossible antecedent are in certain cases true and in other false. I have shown that this interpretation of counterfactuals, today accepted by an increasing number of philosophers, makes Brogaard and Salerno's demonstration invalid and that no amended formulation of the proof compelling for the antirealist appears at hand.

Importantly, the same method used to block Brogaard and Salerno's proof can be employed to reject any of the proofs available today in the literature that aim to show (or that can be read as aiming to show) that global antirealism resting on a counterfactual analysis of truth commits a conditional fallacy. For all these demonstrations require a strict conditional to entail the corresponding counterfactual, and so presuppose that all counterfactuals with impossible antecedent are vacuously true. I refer to Rea (2000: 296)'s proof, Brogaard and Salerno (2005: 125)'s version of Plantinga's proof, Plantinga (1982: 65)'s original proof and a weaker version of the latter due to 
Wright (2000: 342). It is perhaps true that global alethic antirealism resting on a counterfactual analysis of truth commits a conditional fallacy, but no proof of it has yet been delivered.

\section{Acknowledgments}

I am very grateful to Nicola Ciprotti, John Divers, Michael Gabbay, Uriah Kriegel, Jonathan Kvanvig, Julien Murzi, Valeria de Paiva, Tommaso Piazza, Alex K. Simpson, Nicholas J.J. Smith and a referee of this Journal for valuable discussions and important criticisms upon previous versions of this paper. A special thank to Arif Ahmed and Berit Brogaard.

\section{Notes}

${ }^{1}$ Plantinga 1982 and Rea 2000 take the conclusions of their proofs to show that the alethic antirealist is committed to some form of theism (i.e. to the thesis that, roughly, there is necessarily an omniscient epistemic agent/community). Brogaard and Salerno 2005 claim that this is a misguided interpretation of those alleged results, which should instead be seen as instances of the general problem of the conditional fallacy that plagues counterfactual analyses. (The same point is made in Wright 2000). I find this claim straightforward and I will not reconsider it in my paper. ${ }^{2}$ Namely, $\square(\mathrm{T}[P] \leftrightarrow P)$.

${ }^{3}$ By the necessitation of the Equivalence Schema, $(Q)$ 'epistemic conditions are ideal for determining whether [epistemic conditions are ideal for determining whether some statement is true]' entails 'epistemic conditions are ideal for determining whether it is true that [epistemic conditions are ideal for determining whether some statement is true]'. From the latter, it follows: $(P)$ 'epistemic conditions are ideal for determining whether some statement is true'.

${ }^{4}$ Indeed, Brogaard and Salerno 2005 complain that 'Rea unapologetically embraced classical logic and S4, which is highly controversial in this context' (137). This remark - which may be interpreted as questioning axiom 4 - is very puzzling not only because Brogaard and Salerno do not raise any 
similar objection to Plantinga's proof, which presupposes $\mathbf{S 4}$ too, but also because Brogaard and Salerno's own proof is carried out in just an expansion of $\mathbf{S 4}$ ! Berit Brogaard has admitted (in personal communication) that ' $\mathbf{S 4}$ ' is a misprint here, which should be replaced with 'S5'. To strengthen the conclusion of his proof, Rea has in fact appealed to S5 (cf. Brogaard and Salerno 2005: 130).

${ }^{5}$ This claim can be generalized, as the proofs in the literature that aim to show (or that can be read as aiming to show) that the alethic antirealist commits a conditional fallacy are typically carried out in $\mathbf{S 4}$ - so they presuppose axiom 4. (I list these proofs in the conclusion of this paper). The only exception I am aware of is a weaker version of Plantinga's proof due to Wright (2000: 341-342), which aims to demonstrate that Peircean antirealism entails the actual (but not necessary) existence of an omniscient epistemic agent/community. Wright's proof is made in the modal system $\mathbf{T}$ (expanded with resources for counterfactuals), which embeds axiom M but not axiom 4.

${ }^{6}$ A version of Salmon's argument against axiom 4 runs as follows (cf. for instance Salmon 1989: 45, and Hayaki 2005: 28). Axiom 4 is equivalent to $\diamond \diamond P \rightarrow \diamond P$, where $\diamond$ is the possibility operator. Consider an artifact - for example a table, which we will call $\mathrm{T}$. It seems intuitive that $\mathrm{T}$, while retaining its numerical identity, could have originated from a piece of wood $\mathrm{W}_{1}$ very slightly different from the piece of wood $\mathrm{W}_{0}$ from which $\mathrm{T}$ has actually originated. Suppose, for instance, that $\mathrm{W}_{1}$ has the same shape and size as $\mathrm{W}_{0}$ but is taken from one millimeter further down the same tree trunk as $\mathrm{W}_{0}$. This intuition can be expressed by saying, shortly, that it is possible that $\mathrm{T}$ is made of $\mathrm{W}_{1}$. Consider now that if $\mathrm{T}$ had actually originated from $\mathrm{W}_{1}$, it would plausibly be true that $\mathrm{T}$ could have originated from $\mathrm{W}_{2}$, a piece of wood taken from one additional millimeter further down the same trunk. This intuition can be expressed by saying, shortly, that it is possible that it is possible that $\mathrm{T}$ is made of $\mathrm{W}_{2}$. Let us reiterate this reasoning by, say, one thousand times to reach 
the apparently correct conclusion that it is possible that ... it is possible that ... it is possible that $\mathrm{T}$ is made of $\mathrm{W}_{1000}$, namely, a piece of wood that differs from T's actual piece of wood by one meter. As $\diamond \diamond P \rightarrow \diamond P$ entails (by reiterated applications of modus ponens) that any finite string of diamonds can be replaced by just one diamond, from the above claim that it is possible that ... it is possible that ... it is possible that $\mathrm{T}$ is made of $\mathrm{W}_{1000}$, we should derive that it is just possible that $\mathrm{T}$ is made of $\mathrm{W}_{1000}$. But this seems incorrect: if $\mathrm{T}$ had originated from a piece of wood that differs from T's actual piece of wood by one meter, T would plausibly be a distinct individual! This apparent counterexample to axiom 4 might be used by an antirealist who preferred intuitionistic logic to classical logic. I describe systems of intuitionistic modal logic at the end of this section. As $\square$ and $\diamond$ are intuitionistically non-interdefinable, $\square P \rightarrow \square \square P$ does not entail $\diamond \diamond P \rightarrow \diamond P$, and the intuitionistic version of axiom 4 comes in the terms of the conjunction $(\square P \rightarrow \square \square P) \wedge(\diamond \diamond P \rightarrow \diamond P)$. One might perhaps worry that the sorites counterexamples hit only the conjunct $\diamond \diamond P \rightarrow \diamond P$ but not the conjunct $\square P \rightarrow \square \square$, which is actually used in Brogaard and Salerno's proof. But Salmon's argument can easily be formulated to hit directly $\square P \rightarrow \square \square P$.

${ }^{7}$ The fact that when issues of vagueness come into play our intuitions become often confused may cast doubts on the soundness of Salmon's argument. An appropriate evaluation of the latter would probably require a general solution to the problem of vagueness. As Hayaki (2005: 29) suggests, one might be no more convinced by Salmon's argument than by a sorites argument purporting to show that there is no such thing as baldness!

${ }^{8}$ It seems reasonable that $\mathrm{A} 1$ should be accepted if $\mathrm{M}$ is accepted. This is why: for any possible world $w$, if $Q \square \rightarrow R$ is true at $w$, intuitively, $R$ must be true in all possible worlds closest to $w$ in which $Q$ is true (i.e. in all possible worlds that resemble $w$ as much as $Q$ 's truth permits it). Since M entails $Q \rightarrow \diamond Q$, if $\mathrm{M}$ is accepted, any possible world will count as possible to itself. So, if $Q$ is true 
at a world $w, w$ is one of the worlds closest to $w$ at which $Q$ is true. In conclusion, if $\mathrm{M}$ is accepted, for any world $w$, if both $Q \square \rightarrow R$ and $Q$ are true at $w, R$ must also be true at $w$. This seems to validate A1. I do not see how the antirealist could reasonably question this simple and intuitive reasoning.

${ }^{9}$ In this paper, modus ponens is defined in a broadened sense to apply to both $\rightarrow$ and $\leftrightarrow$.

${ }^{10}$ Rigorously speaking, $Q \rightarrow R$ follows from $P \leftrightarrow(Q \square \rightarrow R), Q \rightarrow P$ and axiom A1, used to derive line 5 from lines 3 and 4 . The rule of closure correctly applies here because also A1, as an axiom schema, can be shown to be necessary by necessitation, i.e. the rule that if a statement is a theorem of a given modal logic, it can be necessitated in that logic. A1 represents a theorem of the logic S4 $\cup\{\mathrm{A} 1, \mathrm{~A} 2\}$. As any axiom schema is necessary, for the sake of simplicity I will never mention any of the axioms implicitly appealed to in the applications of the rule of closure made this paper.

${ }^{11}$ These papers provide slightly different axiomatizations of the same intuitionistic modal logics.

${ }^{12}$ Following Simpson (1994: 52), IK can be axiomatized as follows (where $\diamond$ is the possibility operator, $\vee$ is logical disjunction, $\neg$ is logical negation, and $\perp$ is any logical contradiction): Axioms.

(i) All substitution instances of theorems of intuitionistic propositional logic;

(ii) $\quad \square(Q \rightarrow R) \rightarrow(\square Q \rightarrow \square R)$;

(iii) $\square(Q \rightarrow R) \rightarrow(\diamond Q \rightarrow \diamond R)$;

(iv) $\quad \neg \diamond \perp$;

(v) $\quad \nabla(Q \vee R) \rightarrow(\diamond Q \vee \diamond R)$;

(vi) $\quad(\diamond Q \rightarrow \square R) \rightarrow \square(Q \rightarrow R)$.

Rules.

(Modus Ponens) From $Q \rightarrow R$ and $Q$, deduce $R$;

(Necessitation) If $Q$ is a theorem, then so is $\square Q$.

${ }^{13}$ The following two schemata hold valid in the logics of $\mathscr{N}\{\mathbf{I K}\}: \neg \diamond P \leftrightarrow \square \neg P$ and $\diamond \neg P \rightarrow$ $\neg \square P$. Yet $\neg \square P \rightarrow \diamond \neg P$ does not hold valid in them. 
${ }^{14}$ Although this simple and powerful systematisation of intuitionistic modal logic is today accepted by most logicians and computer scientists, weaker and interesting logics have also been studied, often called constructive rather than intuitionistic. See, for instance, de Paiva and Mendler 2005 and Alechina et al. 2001.

${ }^{15}$ The parallelism between the semantics of intuitionistic and classical modal logics is rigorous. Each logic of $\mathscr{I}$ is sound and complete with respect to classes of frames $<\mathrm{W}, \leq, \mathrm{R}>$ in which $\mathrm{R}$ has properties that coincide with those of $\mathrm{R}$ in the classes of frames $<\mathrm{W}, \mathrm{R}>$ with respect to which each parallel logic of $\mathscr{C}$ is sound and complete. Thus, $\mathbf{T}$ and IT are sound and complete with respect to frames in which R is reflexive, S4 and IS4 are sound and complete with respect to frames in which $\mathrm{R}$ is a preorder, and $\mathbf{S 5}$ and IS5 are sound and complete with respect to frames where R is an equivalence relation.

${ }^{16}$ As the intuitionistic $\square$ and $\diamond$ are not dual, the intuitionistic versions of axiom schema $\mathrm{M}$ and axiom schema 4 are, respectively, $(\square P \rightarrow P) \wedge(P \rightarrow \diamond P)$ and $(\square P \rightarrow \square \square P) \wedge(\diamond \diamond P \rightarrow \diamond P)$ (cf. Gabbay et al. 2003: 190 and Simpson 1994: 55-56). For the sake of simplicity, in the logical proofs of this paper I will often use the expressions 'axiom M' and 'axiom 4' to refer to only one of the two conjuncts of, respectively, axiom $\mathrm{M}$ and axiom 4.

${ }^{17}$ To obtain the logic IS4 $\cup\{\mathrm{A} 1, \mathrm{~A} 2\}$, we first expand the alphabet of intuitionistic modal logic with the connective $\square \rightarrow$, and add to the formation rules of the language of intuitionistic modal logic the one such that, if $P$ and $Q$ are well-formed formulae, so is $P \square \rightarrow Q$. Then, we extend the set of axioms of IS4 with A1 and A2. It would be easy to show that the logics of $\mathscr{N}\{\mathbf{I K}\}$ are closed under all elementary inference rules additional to modus ponens used in the proof of CF, and under all those I use in this paper. These elementary rules are: $\rightarrow$ introduction, $\wedge$ introduction, $\wedge$ elimination, and closure. If IS4 is extended to IS4 $\cup\{\mathrm{A} 1, \mathrm{~A} 2\}$, the latter logic will prove closed under the same rules. Since IS4 is logically consistent, IS4 $\cup\{$ A1, A2 $\}$ is also logically consistent. 
Proof. Let us reinterpret $\square \rightarrow$ as $\rightarrow$. As a result, A1 and A2 are turned into, respectively, $(Q \wedge(Q \rightarrow$ $R)) \rightarrow R$ and $\square(Q \rightarrow R) \rightarrow(Q \rightarrow R)$, while there is no change in the inference rules. As both the latter schemata are valid in IS4 (this can easily be shown by using $\rightarrow$ introduction, $\wedge$ introduction, axiom $\mathrm{M}$ and modus ponens) and no formula with the form $P \wedge \neg P$ can be deduced from IS4, no such a formula can be deduced from IS4 $\cup\{$ A1, A2 $\}$ either. QED.

${ }^{18}$ To demonstrate that $\mathbf{I K} \cup\{\mathrm{A} 2\}$ validates $\neg \vee Q \rightarrow(Q \square \rightarrow R)$, let us prove first that $\neg \diamond Q \rightarrow \square(Q$ $\rightarrow R$ ) is valid in IK. Proof. As IK is conservative over intuitionistic propositional logic, IK validates the law of contradiction, i.e. $\neg Q \rightarrow(Q \rightarrow R)$. By necessitation (see above, note 12), we turn $\neg Q \rightarrow(Q \rightarrow R)$ into $\square(\neg Q \rightarrow(Q \rightarrow R))$. This schema and axiom (ii), i.e. $\square(Q \rightarrow R) \rightarrow(\square Q \rightarrow$ $\square R$ ) (see ibid.), entail $\square \neg Q \rightarrow \square(Q \rightarrow R)$ by modus ponens. IK validates the schema $\neg \vee Q \rightarrow \square \neg Q$ (see above, note 13). Assume $\neg \vee Q$ for $\rightarrow$ introduction. $\neg \vee Q$ and $\neg \vee Q \rightarrow \square \neg Q$ entail $\square \neg Q$ by modus ponens. $\square \neg Q$ and $\square \neg Q \rightarrow \square(Q \rightarrow R)$, derived before, entail $\square(Q \rightarrow R)$ by modus ponens. Finally, $\neg \diamond Q$, assumed above, and $\square(Q \rightarrow R)$ entail $\neg \diamond Q \rightarrow \square(Q \rightarrow R)$ by $\rightarrow$ introduction. QED. Let us now demonstrate that IK $\cup\{\mathrm{A} 2\}$ validates $\neg \diamond Q \rightarrow(Q \square \rightarrow R)$. Proof. Assume $\neg \diamond Q$ for $\rightarrow$ introduction. This assumption and the schema $\neg \vee Q \rightarrow \square(Q \rightarrow R)$, which has been shown to hold valid in IK, entail $\square(Q \rightarrow R)$ by modus ponens. $\square(Q \rightarrow R)$ and A2 - i.e. $\square(Q \rightarrow R) \rightarrow(Q \square \rightarrow R)-$ entail $Q \square \rightarrow R$ by modus ponens. Finally, $\neg \diamond Q$, assumed initially, and $Q \square \rightarrow R$ entail $\neg \diamond Q \rightarrow(Q$ $\square \rightarrow R$ ) by $\rightarrow$ introduction. QED.

${ }^{19}$ Consider any possible world $w$. If $\diamond Q$ is true at $w$, there are worlds that are possible to $w$ and in which $Q$ is true. If $\square(Q \rightarrow R)$ is also true at $w$, in all these worlds - and thus in the closest to $w-R$ is true. Given Lewis' only clause (b), $Q \square \rightarrow R$ is also true at $w$. This seems to validate A3. There is no apparent reason why the antirealist should reject this intuitive reasoning.

${ }^{20}$ More precisely, as the intuitionistic $\square$ and $\diamond$ are not dual, axiom 5 is to be formulated as follows: $(\diamond Q \rightarrow \square \diamond Q) \wedge(\diamond \square Q \rightarrow \square Q)$ (cf. Gabbay et al. 2003: 190, and Simpson 1994: 55-56). 
${ }^{21}$ Although I will not pursue this possible line of defence of antirealism here, it is worth emphasizing that the difficulties that the antirealist might raise against the adequacy of axiom 4 - as I have suggested in Sect 2 - would count against IS5 too, as the latter validates the former. ${ }^{22}$ The following sketched argument, based on the version of combinatorialism presented in Divers (2002: 174-176 and 207-208) appears to strike axiom 5 independently of whether classical or intuitionistic logic is presupposed. For the combinatorialist, a state of affairs is possible if and only if it can be obtained by recombining actually existing simple individuals and actually instantiated simple properties. Simple individuals are those that lack proper parts, and simple properties are those that do not have other properties as constituents. Simple individuals and simple properties exist only contingently. (To simplify, I assume that the actual states of affairs represent a limiting case of recombination). Consider any simple property $\mathrm{P}$ actually instantiated by some individual and any existing simple individual a. The state of affairs that $\mathrm{Pa}$ can be obtained by recombining a and $\mathrm{P}$. Therefore, it holds true that $\diamond \mathrm{Pa}$. Consider now all recombinations of the actually instantiated property $\mathrm{P}$ with all existing simple individuals: one recombination is to the effect that $\mathrm{P}$ is instantiated by no individual at all. This constitutes a genuine possibility for the combinatorialist something that could have happened. But, in that case, if $\mathrm{P}$ had been instantiated by no individual at all, it would not have been possible that $\mathrm{Pa}$, for there would have been no existing $\mathrm{P}$ to be recombined with the individual a in the state of affairs that $\mathrm{Pa}$. Therefore, although it is true that $\diamond \mathrm{Pa}$, it is also possible that $\neg \diamond \mathrm{Pa}-$ briefly, it is also true that $\diamond \neg \diamond \mathrm{Pa}$. As both $\mathbf{K}$ and IK validate the schema $\diamond \neg P \rightarrow \neg \square P, \diamond \neg \diamond \mathrm{Pa}$ entails $\neg \square \diamond \mathrm{Pa}$ in both classical and intuitionistic modal logic. In conclusion, for any simple property $\mathrm{P}$ actually instantiated and any existing simple individual a, it turns out that $\diamond \mathrm{Pa} \wedge \neg \square \diamond \mathrm{Pa}$. This is in plain contradiction with axiom 5. The combinatorialist cannot accept this axiom. 
${ }^{23}$ For a critical overview of the principal theories of possibility including combinatorialism, see for instance Divers 2002.

${ }^{24}$ It can easily be shown that, in IS4 $\cup\{\mathrm{A} 3\}$ (and so in IS4 $\cup\{\mathrm{A} 1, \mathrm{~A} 3\}$ ), A3 is equivalent to (A3*) $\diamond Q \rightarrow(\square(Q \rightarrow R) \rightarrow(Q \square \rightarrow R))$. Appealing to A3* rather than A3 in the derivation of $Q \square \rightarrow R$, on line 20, produces no improvement. In this case, $\square(Q \square \rightarrow R)$ should be derived from $\square \square(Q \rightarrow R)$ and $\square(Q \rightarrow R) \rightarrow(Q \square \rightarrow R)$, by closure. The problem is that only the first schema but not the second is necessary.

${ }^{25}$ It would be easy to show that, in IS4 $\cup\{\mathrm{A} 4\}$ (and so in IS4 $\cup\{\mathrm{A} 1, \mathrm{~A} 4\}$ ), A4 is equivalent to $(\square(Q \rightarrow R) \wedge \diamond Q) \rightarrow \square(Q \square \rightarrow R)$.

${ }^{26}$ Proof. Suppose CF2 is demonstrable in IS4 $\cup\{\mathrm{A} 1\}$. Let us substitute, in CF2, $P$ for $Q$, a tautology $\mathrm{T}$ for $R$, and $\leftrightarrow$ for $\square \rightarrow$. The resulting meta-statement is:

(1) $\{\square(P \leftrightarrow(P \leftrightarrow \mathrm{T})), \square(P \rightarrow P), \square(P \rightarrow \mathrm{T}) \rightarrow(P \leftrightarrow \mathrm{T})\}$ entails $\square P$.

If CF2 were provable in IS4 $\cup\{\mathrm{A} 1\}$, (1) should be provable in IS4 (since $\leftrightarrow$ obeys modus ponens, which is a rule of IS4, and modus ponens is also the only principle that IS4 $\cup\{\mathrm{A} 1\}$ specifies for $\square \rightarrow)$. As IK is conservative over intuitionistic propositional logic, IK, and so IS4, validates both $P$ $\leftrightarrow(P \leftrightarrow \mathrm{T})$ and $P \rightarrow P$ and, by necessitation, both $\square(P \leftrightarrow(P \leftrightarrow \mathrm{T}))$ and $\square(P \rightarrow P)$. Consequently, (1) can be simplified into:

(2) $\square(P \rightarrow \mathrm{T}) \rightarrow(P \leftrightarrow \mathrm{T})$ entails $\square P$.

If CF2 were provable in IS4 $\cup\{\mathrm{A} 1\}$, (2) should be provable in IS4. Yet, as IS4 is conservative over intuitionistic propositional logic, IS4 validates $P \rightarrow(P \leftrightarrow \mathrm{T})$. By this schema, modus ponens and $\rightarrow$ introduction, it is possible to show that the following meta-statement is provable in IS4:

(3) $P$ entails $\square(P \rightarrow \mathrm{T}) \rightarrow(P \leftrightarrow \mathrm{T})$. 
As a result, if (2) were provable in IS4, through (3) this would yield a proof of $\square P$ from $P$, which is known to be invalid in IS4. Since (2) is not provable in IS4, (1) is not provable in IS4 $\cup\{$ A1 $\}$. QED. I am grateful to a referee of this journal for suggesting this proof.

${ }^{27}$ It would be easy to show that, in IS4 $\cup\{\mathrm{A} 1\}$, the schema $\square(\square(Q \rightarrow R) \rightarrow(Q \square \rightarrow R))$ is equivalent to the schema $\square(Q \rightarrow R) \rightarrow \square(Q \square \rightarrow R)$.

${ }^{28}$ For instance, IS4 $\cup\{\mathrm{A} 1, \mathrm{~A} 3\}$ could be supplemented with some of the rules and principles for counterfactuals assumed by Lewis (cf. 1973: 132), provided that the logics so obtained do not validate the questionable axiom schema $\mathrm{A} 2$.

${ }^{29}$ Indeed, the other proofs available in the literature aiming to show (or that can be read as aiming to show) that the alethic antirealist commits a conditional fallacy (see the conclusion of this article) assume no axiom schema specific for counterfactuals other than A1 and the problematic A2 (or the corresponding inference rules).

\section{References}

Alechina, N. and V. De Paiva, M. Mendler, E. Ritter. (2001). Categorical and Kripke Semantics for Constructive S4 Modal Logic. In L. Fribourg (ed.), Proceeding of Computer Science Logic (CSL'01), LNCS 2142.

Armstrong, D. M. 1989. A Combinatorial Theory of Possibility. Cambridge: Cambridge University Press.

Brogaard, B. and J. Salerno. 2005. 'Anti-realism, Theism and the Conditional Fallacy'. Nous 39: 123-139.

De Paiva, V. and M. Mendler. 2005. 'Constructive CK for contexts'. Proceeding of the Workshop on Context Representation and Reasoning, Paris, France, July 2005.

Divers, J. 2002. Possible Worlds. London: Routledge. 
Fisher Servi, G. 1980. 'Semantics for a class of intuitionistic modal calculi'. In M. L. Dalla Chiara (ed.), Italian studies in the Philosophy of Science: 59-72. Dordrecht: Reidel.

Fisher Servi, G. 1984. ‘Axiomatizations for some intuitionistic modal logics'. Rendiconti di Matematica di Torino 42: 179-195.

Gabbay, D. and A. Kurucz, F. Wolter, M. Zakharyaschev. 2003. Many-Dimensional Modal Logics: Theory and Applications. Studies in Logic. Amsterdam: Elsevier.

Goodman, J. 2004. 'An Extended Lewis/Stalnaker Semantics and the New Problem of Counterpossibles'. Philosophical Papers 33: 35-66.

Hayaki, R. 2005. 'The Transience of Possibility'. European Journal of Analytic Philosophy 1(2): 25-36.

Lewis, D. K. 1973. Counterfactuals. Cambridge: Harvard University Press.

Mares, E. D. 1997. 'Who’s Afraid of Impossible Worlds?'. Notre Dame Journal of Formal Logic 38: 516-526.

Nolan, D. 1997. 'Impossible Worlds: A Modest Approach'. Notre Dame Journal of Formal Logic 38: $535-572$.

Plantinga, A. 1982. 'How to be an Anti-realist'. Proceedings and Addresses of the American Philosophical Association 56: 47-70.

Priest, G. 1997. 'Sylvan's Box’. Notre Dame Journal of Formal Logic 38: 573-582.

Rea, M. 2000. 'Theism and Epistemic Truth-Equivalences'. Nous 34: 291-301.

Read, S. 1995. Thinking about Logic. Oxford: Oxford University Press.

Salmon, N. 1989. 'The logic of what might have been'. Philosophical Review 98: 3-34.

Salmon, N. 1993. 'This side of paradox'. Philosophical Topics 21: 187-197.

Simpson, A. K. 1994. The Proof Theory and Semantics of Intuitionistic Modal Logic. PhD Thesis. University of Edinburgh. 
Wright, C. 2000. 'Truth as Sort of Epistemic: Putnam's Peregrinations'. Journal of Philosophy 97: $335-364$.

Yagisawa, T. 1988. 'Beyond Possible Worlds'. Philosophical Studies 53: 175-204.

Zagzebski, L. 1990. ‘What if the Impossible Had Been Actual?’ In M. Beatty (ed.), Christian Theism and the Problems of Philosophy. Notre Dame: University of Notre Dame Press. 